\title{
Effects of auditory interference upon observed lingual tactile thresholds
}

\author{
KAL M. TELAGE and JANET C. SCOTT \\ Department of Speech Pathology and Audiology, Ithaca College, Ithaca, New York 14850
}

\begin{abstract}
Lingual vibrotactile thresholds were obtained from 16 adult subjects equally divided into experimental and control groups. The experimental subjects were required to read for $3 \mathrm{~min}$ under an auditory masking condition. Control subjects experienced 3 min of silence. Pre- and postexperimental lingual vibrotactile thresholds did not differ significantly between groups. Although this finding differed from previous research, it appeared to be consistent with response characteristics of the neural transducers that were stimulated.
\end{abstract}

Development of lingual vibrotactile instrumentation by Fucci in 1969 sparked interest in the use of lingual vibrotactile thresholds to further investigate Fairbanks' (1954) theoretical model of speech as a servosystem. Prior to that time, the role of lingual tactile feedback in the regulation of speech production had primarily been investigated by using oral stereognosis as a measure of oral sensory function (Bloomer, 1967; Bosma, Grossman, \& Kavanagh, 1967; Chase, 1967; Ringel, Burk, \& Scott, 1968). Lingual vibrotactile threshold testing eventually allowed the examiner stimulus control over such stimulus parameters as frequency, intensity, and duration (Telage \& Fucci, 1973). Recent studies using oral vibrotactile stimulation have attempted to evaluate interactions between auditory and tactile sense modalities.

Fucci, Crary, Warren, and Bond (1977) employed lingual vibrotactile threshold testing to further investigate Sussman's (1970) theory of "a sensitive auditorytactual synchronization system for speech motor activities" (p.320). These authors obtained lingual vibrotactile thresholds for subjects under experimental and control conditions. Experimental subjects received 80-dB HTL of wide-band noise while simultaneously reading selected speech segments for $3 \mathrm{~min}$. The control subjects read the segments without exposure to noise. Results revealed significantly reduced sensitivity (.05) between pre- and posttest lingual vibrotactile thresholds in the experimental group. No such difference was found in the control group. It was felt that the experimental variable had interfered with lingual tactile sensitivity.

Crary, Fucci, and Bond (1979) investigated the effect of exposure to 90-dB SPL of babble noise on lingual vibrotactile sensitivity. Subjects read prose passages for various intervals ranging from 30 to $180 \mathrm{sec}$ while exposed to this noise. The authors noted that increased exposure to noise was correlated with reduced lingual tactile sensitivity. These findings further supported Sussman's (1970) view of a sensory feedback interaction.

The purpose of the present investigation was to further test Sussman's (1970) theory using a modified but similar vibrotactile testing paradigm. The primary methodological difference is that the present procedure isolates neural receptors in the anterior dorsal lingual surface from more ventral receptors. This is achieved by testing the tongue in an unclamped posture while using an upper static-surround disk.

\section{METHOD}

\section{Subjects}

Sixteen adult subjects, ranging in age from 18 to 28 years, were randomly selected from a pool of 50 college students and randomly divided into a control and an experimental group. No subjects reported a history of a speech disorder or sensorimotor impairment.

Before testing, subjects were given several training trials to familiarize themselves with the nature of the stimulus and the task. Standardized written instructions were read to each subject during the training phase. Nine threshold responses were obtained at a frequency of $250 \mathrm{~Hz}$, using a descending psychophysical method of limits. A training criterion required each subject to produce two of the last three threshold responses within $10 \mathrm{mV}$ of each other. Upon meeting this criterion, subjects immediately began the test phase.

\section{Apparatus}

Figure 1 presents a block diagram of the instrument package used in this study. The stimulus control unit is composed of Coulbourn solid state logic modules. These units generate pulsed vibratory signals that may be varied in frequency, intensity, and temporal characteristics. Three universal timers are programmed to control signal duration and duty cycle. The timers gate a selectable rise-fall module on and off. This continuously adjustable electronic switch was set to generate a rise-fall time of $100 \mathrm{msec}$. The signal from the rise-fall module was fed into a precision signal generator and an audio mixer amplifier. Stimulus intensities were varied in increments of $256.5-\mathrm{dB}$ steps by passing them through a programmable attenuator and an 8-bit binary up-down counter. Pulsed signals from the stimulus control unit drive the electromagnetic minivibrator that is the stimulus-producing aggregate of the system.

Figure 2 presents a schematic diagram of the vibrator and modified clamp assembly portion of the vibrotactile stimulator. The lower clamping arm and its disk were removed. The upper clamping arm with a free-surround disk was retained. All contact points between the vibrator and cowling were insulated by rubber stripping.

The vibrator probe with attached contactor was lowered 


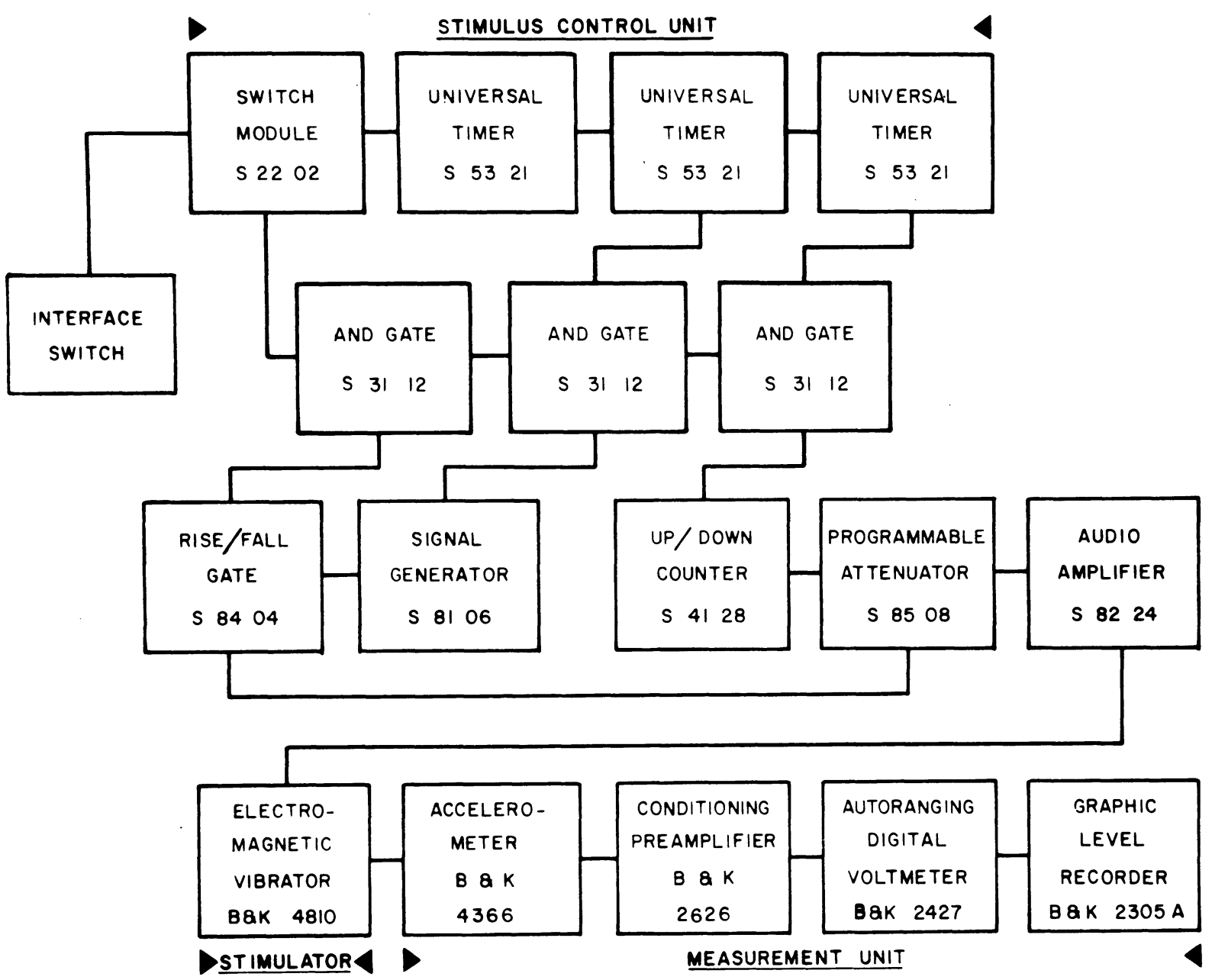

Figure 1. A block diagram of the automated instrumentation system.

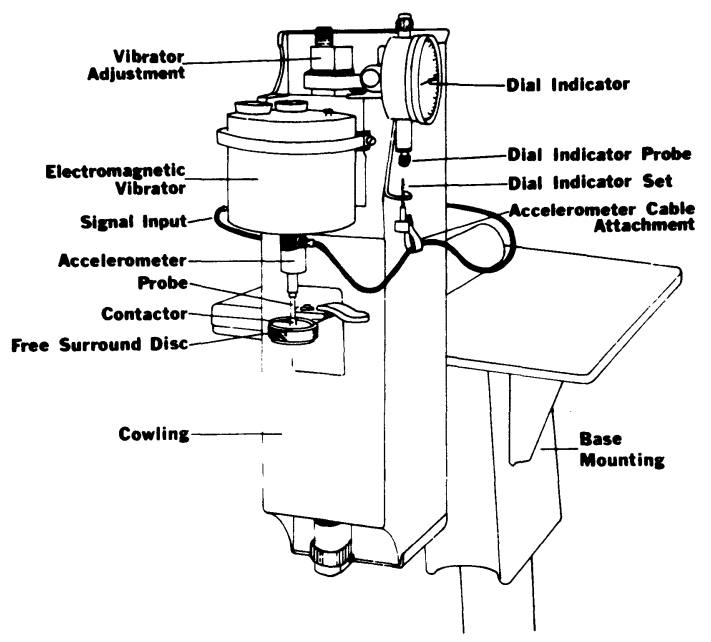

Figure 2. Schematic diagrams of the vibrator, free-surround, and contactor adjustment aspects of the oral vibrotactile stimulator.
$1 \mathrm{~mm}$ below the lower surface of the free-surround disk. The area of the contactor was $.128 \mathrm{~cm}^{2}$. The diameter of the opening in the free-surround disk was $2 \mathrm{~mm}$ larger than the diameter of the contactor.

An accelerometer mounted on the probe measured contactor displacement as a small voltage. Displacement voltages were directly amplified by a conditioning preamplifier that was set to emit $100 \mathrm{mV} / \mathrm{g}$ of acceleration. Signals were constantly monitored by an autoranging digital voltmeter set to read peak displacement values in millivolts.

A wide-band noise generator provided auditory masking at 70 and $90 \mathrm{~dB}$ SPL through 7DH-39 headphones.

\section{Procedure}

Standardized instructions were read to each subject during the test phase. With headphones on, each subject was comfortably seated in an adjustable chair. Subjects were instructed to hold the upper surface of the tongue against a free-surround disk and to indicate with a finger when the stimulus was detected.

The test phase, lasting $6 \mathrm{~min}$, consisted of a pretest and posttest separated by a 1 -min break and either $3 \mathrm{~min}$ of silence (control group) or $3 \mathrm{~min}$ of reading under exposure to $90-\mathrm{dB}$ SPL of wide-band noise (experimental group). During both the pretest and posttest, three lingual vibrotactile thresholds were 
obtained at $250 \mathrm{~Hz}$ with 70-dB SPL of broad-band masking.

Median displacement values in peak millivolts were accepted as threshold. The data, initially recorded in millivolts, were subsequently converted to microns of peak displacement using an acceleration formula.

\section{RESULTS AND DISCUSSION}

Table 1 presents pretest and posttest threshold data in microns, averaged across subjects in each group. A $t$ test for independent samples revealed no significant difference between the threshold changes in the two groups. It appears on the basis of these findings that the experimental condition of masking during speech did not alter oral tactile sensitivity.

It is interesting to speculate about possible reasons for the difference between these results and those of Crary and his associates. This discrepancy might be explained by the difference in neural receptor populations resulting from clamping the tongue. It has previously been shown that the receptors on the lingual dorsal surface differ from the more ventrally located Pacinian corpuscles in such response characteristics as frequency-sensitivity (Telage \& Petrosino, 1978) and spatial summation (Telage \& Warren, 1977). Additionally, clamping the tongue tends to push the Pacinian receptors toward the surface, where they may be more easily excited by vibratory stimulation. It is possible that these two classes of mechanoreceptors are distinctly different in terms of their interaction with the experimental variables.

Although our results differ from the findings of Crary et al. (1979) and Fucci et al. (1977) regarding the effects of masking and speech on lingual tactile sensitivity, they do not necessarily conflict with Sussman's (1970) view of balanced auditory-tactile interactions. Rather, they might provide a more accurate physiological perspective concerning the nature of the tactile transducers that act in synchrony with the auditory system. Clearly, there appear to be two distinct mechanisms that provide oral tactile feedback. One mechanism, the Pacinian corpuscle, summates energy spatially and temporally in a manner not unlike the auditory system (Zwislocki, 1960). It is quite reasonable to theorize that this tactile system works in concert with audition in providing balanced sensory input for complex motor synergies. On the other hand, our findings would strongly suggest that mechanoreceptors found on the lingual surface are a more independent afferent mechanism. Further research
Table 1

Means for Lingual Vibrotactile Threshold Data in Microns of Peak Displacement

\begin{tabular}{lcc}
\hline & Experimental & Control \\
\hline Pretest & 1.67 & 1.51 \\
Posttest & 1.58 & 1.38 \\
Difference & $.09^{*}$ & $.02^{*}$ \\
\hline
\end{tabular}

*Nonsignificant.

into the nature of these receptors might better delineate their functional characteristics.

\section{REFERENCES}

Bloomer, H. Oral manifestations of diadochokinesis with oral astereognosia. In J. Bosma (Ed.), Symposium on oral sensation and perception. Springfield, Ill: Thomas, 1967.

Bosma, J., Grossman, R., \& Kavanagh, J. A syndrome of impairment of oral perception. In J. Bosma (Ed.), Symposium on oral perception. Springfield, Ill: Thomas, 1967.

Chase, R. Abnormalities in motor control secondary to congenital sensory deficits. In J. Bosma (Ed.), Symposium on oral sensation and perception. Springfield, Ill: Thomas, 1967.

Crary, M., Fucci, D., \& Bond, Z. Timed auditory interruption during speech: Oral sensory and temporal articulatory changes. Journal of Phonetics, 1977, 7, 335-342.

Fairbanks, G. Systematic research in experimental phonetics: I. A theory of the speech mechanism as a servosystem. Journal of Speech and Hearing Disabilities, 1954, 19, 133-139.

Fucci, D. Oral vibrotactile perception: An evaluation of normal and defective speakers. Unpublished doctoral dissertation, Purdue University, 1969.

Fucci, D., Crary, M., Warren, J., \& Bond, Z. Interaction between auditory and oral sensory feedback in speech regulation. Perceptual and Motor Skills, 1977, 45, 123-129.

Ringel, R., Burk, K., \& Scott, C. Tactile perception: Form discrimination in the mouth. British Journal of Communication Disorders, 1968, 3, 150-155.

Sussman, H. The role of sensory feedback in tongue movement control. Journal of Auditory Research, 1970, 10, 296-320.

Telage, K., \& Fucci, D. Vibrotactile stimulation: A future clinical tool for speech pathologists. Journal of Speech and Hearing Disabilities, 1973, 38, 442-447.

Telage, K., \& Petrosino, L. Testing procedures for measuring oral vibrotactile thresholds: III. Effects obtained using a nonclamping method. Bulletin of the Psychonomic Society, 1978, 12, 291-293.

Telage, K., \& Warren, J. Spatial summation effects on lingual vibrotactile thresholds. Perceptual and Motor Skills, 1977, 44, 1179-1185.

Zwislocki, J. A theory of temporal summation. Journal of the Acoustical Society of America, 1960, 32, 1046-1060.

(Received for publication April 15, 1980.) 